\title{
Theoretical Predictions of Lattice Parameters and Mechanical Properties of Pentaerythritol Tetranitrate under the Temperature and Pressure by Molecular Dynamics Simulations
}

\author{
J.J. TAN ${ }^{a, b, c}$, C.E. HU ${ }^{d, *}$, Y. $\mathrm{LI}^{b}$, N.N. GE ${ }^{c}$, T. CHEN ${ }^{b}$ AND G.F. JI ${ }^{c}$ \\ ${ }^{a}$ State Key Laboratory Breeding Base of Nuclear Resources and Environment, East China University of Technology, \\ Nanchang, 330013, China \\ ${ }^{b}$ College of Science, East China University of Technology, Nanchang, 330013, China \\ ${ }^{c}$ Laboratory for Shock Wave and Detonation Physics Research, Institute of Fluid Physics, \\ Chinese Academy of Engineering Physics, Mianyang, 621900, China \\ ${ }^{d}$ College of Physics and Electronic Engineering, Chongqing Normal University, Chongqing, 400047, China
}

(Received March 16, 2016; in final form January 17, 2017)

\begin{abstract}
Molecular dynamics simulations with condensed-phase optimized molecular potentials for atomistic simulation studies force field are performed to investigate the structure, equation of state, and mechanical properties of high energetic material pentaerythritol tetranitrate. The equilibrium structural parameters, pressure-volume relationship and elastic constants at ambient conditions agree excellently with experiments. In addition, fitting the pressure-volume data to the Birch-Murnaghan or Murnaghan equation of state, the bulk modulus $B_{0}$ and its first pressure derivative $B_{0}^{\prime}$ are obtained. Moreover, the elastic constants are calculated in the pressure range of 0-10 GPa at room temperature and in the temperature range of 200-400 K at the standard pressure, respectively. By the Voigt-Reuss-Hill approximation, the mechanical properties such as bulk modulus $B$, shear modulus $G$, and the Young modulus $E$ are also obtained successfully. The predicted physical properties under temperature and pressure can provide powerful guidelines for the engineering application and further experimental investigations.
\end{abstract}

DOI: 10.12693/APhysPolA.131.318

PACS/topics: molecular dynamics, lattice parameter, equation of state, elasticity, mechanical properties

\section{Introduction}

Pentaerythritol tetranitrate (PETN), as one of the most powerful explosive materials, has received considerable attention because of its wide applications in industrial and military fields. In the past decades, many researches on the equation of state (EOS) and mechanical properties have been done for PETN by theory and experiments [1-10]. Accurate EOS and elastic constants are of particular importance because they provide an excellent opportunity to link the microscopic and macroscopic properties of PETN on atomic and molecular scale [1].

At ambient conditions, the crystalline PETN exists as PETN-I, whose crystal structure belongs to the tetragonal symmetry [2]. The phase transition of PETN-I occurs to PETN-II at high temperature, and the PETNII belongs to the orthorhombic symmetry [3]. The safety problems of high energy density material like PETN are always important. The temperature and pressure are the influencing factors in preparation, transportation, storage, and handing process. To investigate the EOS of PETN is of key importance in theory or experi-

*corresponding author; e-mail: cuiehu@126.com ment. Olinger et al. measured the isothermal linear and volume compressions of PETN up to $10 \mathrm{GPa}$ by a high pressure X-ray diffraction technique [2]. Subsequently, considerable effort has been done to simulate the EOS of PETN. Although the density functional theory (DFT) is successful in predicting the properties of many materials, it cannot give accurate descriptions for the molecular crystal PETN because of the poor description on long-range behavior in DFT. Using the Perdew-BurkeErnzerhof (PBE) functional, the projected augmented wave (PAW) function pseudopotential has been employed to calculate the crystal structures of PETN under pressure with $545 \mathrm{eV} \mathrm{[4]} \mathrm{or} 700 \mathrm{eV}$ [1] plane-wave basis set, respectively. There are significant discrepancies in crystal volume at zero pressure between the results from DFT and the experiments. But the discrepancy becomes smaller with increasing pressure. The discrepancy of the lattice volume at zero pressure with $700 \mathrm{eV}$ is much larger than that of $545 \mathrm{eV}[1,4]$. This phenomenon also appears in other DFT calculations, such as ultrasoft pseudopotentials (USP) within generalized gradient approximation (GGA) Perdew-Wang 91 (PW91) or local density approximation (LDA) functional [4]. The reason is that the DFT cannot give good description of the long-range nonbond interactions. In contrast, the calculated zero pressure lattice parameters of PETN by both of the molecular simulations within the rigid-molecule approximation 
and molecular packing method are accurate, but deviations have been getting larger with increase of pressure in comparison with the experimental results. The similarity of the results indicates that both of the two methods cannot predict high pressure properties accurately [5]. The condensed-phase optimized molecular potentials for atomistic simulation studies (COMPASS) force field, a high quality general force field, could effectively improve the accuracy of the simulations for energetic materials [6]. Because the COMPASS force field gives the well description of the intermolecular interaction potential. Here, we employ the MD simulations with COMPASS force field to investigate the lattice dynamics of PETN.

The chemical decomposition and mechanical deformation are dependent on the intermolecular interactions, molecular arrangements, and molecular composition $[7,8]$. The elastic constant is a key parameter to investigate the mechanism of a detonation initiation and mechanical deformation. Winey and Gupta have used ultrasonic measurements to determine the elastic constants of PETN [9]. Subsequently, Sun have measured elastic constants of PETN using impulsive stimulated thermal scattering [10]. Theoretically, the elastic constants $\left(C_{11}\right.$, $C_{22}$, and $C_{33}$ ) have been obtained by first-principles DFT calculations [1]. Zaoui and Sekkal have calculated the elastic constants $\left(C_{11}, C_{12}\right.$, and $\left.C_{44}\right)$ using MD simulation based on a three-body potential [11]. The calculated elastic constants are in agreement with experimental data. All the data of elastic constants above are obtained at ambient condition [9-11]. The elastic constants of PETN under high pressure and temperature are scarce.

As described above, the EOS and elastic constants of PETN have been studied. Our primary goal of this study is to investigate the influence of temperature and pressure on the lattice parameters and mechanical properties. The calculated data can provide practical guidance for engineering application.

\section{Methods and calculation details}

The MD simulations with COMPASS [6] force field are employed to calculate the properties of PETN-I and PETN-II. The initial crystal lattice parameters and internal atomic coordinates PETN-I [12] and PETN-II [3] are taken from experiments. PETN is a molecular crystal with the molecular formula $\mathrm{C}_{5} \mathrm{H}_{8} \mathrm{~N}_{4} \mathrm{O}_{12}$. PETN-I has two molecules per unit cell and belongs to the tetragonal symmetry. PETN-II has four molecules per unit cell and belongs to the orthorhombic symmetry. PETN-I and PETN-II, together with the conformation and atomic numbering of $\mathrm{C}_{5} \mathrm{H}_{8} \mathrm{~N}_{4} \mathrm{O}_{12}$ molecule are plotted in Fig. 1. The MD simulations are performed with the Discover code [13]. The MD simulations were carried out in the isothermal-isobaric NPT ensemble with the Berendsen barostat method [14] and the Andersen thermostat method [15] to control the system pressures and temperatures. The non-bond interactions are managed using the Ewald summation method [16]. The MD simulations are done with a computational supercell size of $3 \times 3 \times 3$ unit cells. At each target of temperature and pressure, the system is relaxed for $1 \times 10^{5}$ time steps with the time step of $1 \mathrm{fs}$ in the equilibration run in all MD simulations. The energy and temperature versus simulation time of PETN I at ambient conditions are shown in Fig. 2. (a)

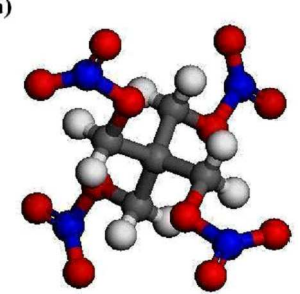

(c)

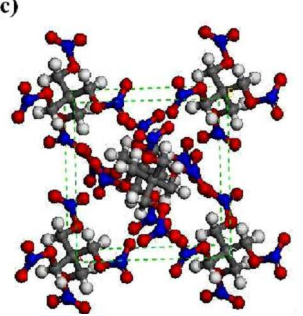

(b)
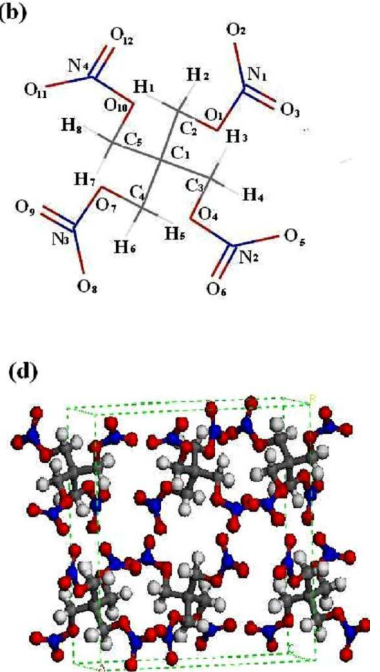

Fig. 1. Conformation (a) and atomic numbering (b) of $\mathrm{C}_{5} \mathrm{H}_{8} \mathrm{~N}_{4} \mathrm{O}_{12}$ molecule in PETN unit cell of PETN-I (c) and PETN-II (d).

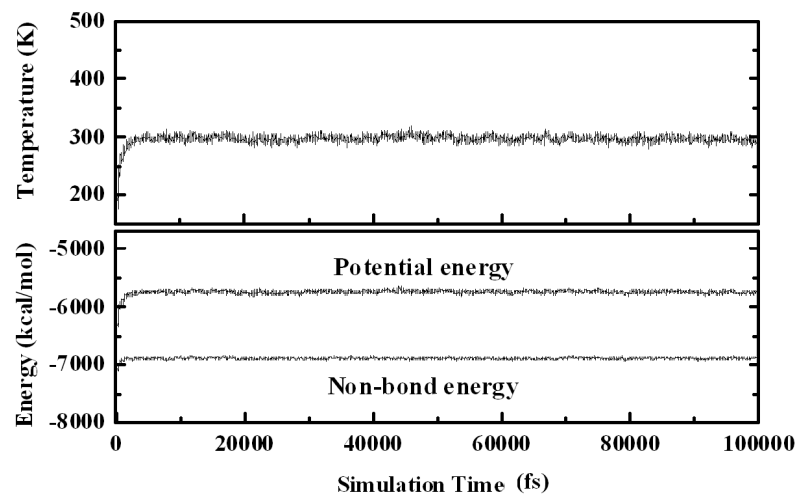

Fig. 2. The energy and temperature versus simulation time of PETN-I at ambient conditions.

After the MD simulation has been done, the resulting equilibrium structure is analyzed to determine elastic constants. The elastic constants of the final atomic configuration are computed using the static approach. The elastic stiffness tensor $C_{i j k l}$ can be expressed as

$$
C_{i j k l}=\frac{\partial \sigma_{i j}}{\partial \sigma_{k l}}\left|T, \varepsilon_{k l}=\frac{1}{V_{0}} \frac{\partial^{2} E}{\partial \varepsilon_{i j} \partial \varepsilon_{k l}}\right| T, \varepsilon_{i j}, \varepsilon_{k l},
$$

where the $C_{i j k l}$ is related to the stress tensor $\sigma$, the strain tensor $\varepsilon$. The $E$ and $V_{0}$ represent the Helmholtz free energy and volume of the equilibrium cell in the undeformed configuration, respectively. Due to the symmetry of 
the stress and strains, and the conservation of energy, the fourth-order $C_{i j k l}$ may be reduced to the second-order tensor. Generally speaking, the elastic stiffness tensor has 21 non-zero independent components. However, this number is greatly reduced when taking the symmetry of the crystal into account. As is known, there are six independent components of the elastic stiffness tensor for tetragonal crystal, i.e. $C_{11}, C_{12}, C_{13}, C_{33}, C_{44}, C_{66}$ and nine independent components for orthorhombic crystal, i.e. $C_{11}, C_{22}, C_{33}, C_{44}, C_{55}, C_{66}, C_{12}, C_{13}$, and $C_{23}$.

\section{Results and discussion}

\subsection{Lattice parameters}

Determination of the equilibrium parameters of PETN crystals is important not only for the knowledge of the molecular crystal structure at ambient condition, but also for benchmarking the performance of the theoretical approach to describe molecular and intermolecular interaction. The calculated lattice constants and unit cell volume at ambient conditions are shown in Table I, together with the available experimental [2, 3] and other theoretical data $[1,5,17,18]$. Using norm-conserving pseudopotentials, the DFT method with a cutoff of $1200 \mathrm{eV}$ for the plane-wave basis, is used to calculate the cell parameters [17]. Because there are no corrections for the van der Waals interaction, the CASTEP calculations significantly overshoot the cell volume, with the deviation of $14.22 \%$ [17]. The calculations within projected augmented wave function pseudopotential with $700 \mathrm{eV}$ [1] plane wave basis set overestimate the cell volume, with the deviation of $6.88 \%$. In the all-electron calculations, both the STO-3G basis set and the $6-31 \mathrm{G}^{* *}$ Gaussian basis set seem to well reproduce the experimental cell volume, with the deviation of $-3.21 \%$ [18] and $2.04 \%$ [17], respectively. In addition, the MD simulations within the rigid-molecule approximation and molecular packing calculations achieve good agreement with experimental data at ambient conditions. Our calculated NPT-MD results agree well with the experiments and the calculated results $[2,3]$. The accuracy of the simulations for energetic materials could be effectively improved by the advanced COMPASS force field, which gives good description of the intermolecular interaction potential. The quantitative descriptions of the lattice parameters as a function of pressure (for $P \leq 10 \mathrm{GPa}$ ) are

$$
\begin{aligned}
& V_{\text {PETN-I }}=576.4750-37.3547 P+4.4280 P^{2} \\
& \quad-0.2099 P^{3}, \\
& a_{\text {PETN-I }}=9.3139-0.2088 P+0.0238 P^{2} \\
& \quad-0.0011 P^{3}, \\
& c_{\text {PETN-I }}=6.6482-0.1459 P+0.0164 P^{2} \\
& \quad-7.7477 \times 10^{-4} P^{3}, \\
& V_{\text {PETN-II }}=1167.1493-82.6185 P+10.4880 P^{2} \\
& \quad-0.5001 P^{3}, \\
& a_{\text {PETN-II }}=13.0830-0.3238 P+0.0401 P^{2}
\end{aligned}
$$

$$
\begin{aligned}
& -0.0019 P^{3}, \\
& b_{\text {PETN-II }}=13.2739-0.3235 P+0.0398 P^{2} \\
& \quad-0.0019 P^{3}, \\
& c_{\text {PETN-II }}=6.7239-0.1637 P+0.0200 P^{2} \\
& \quad-9.5125 \times 10^{-4} P^{3} .
\end{aligned}
$$

The quantitative descriptions of the lattice parameters as a function of temperature for $200 \mathrm{~K} \leq T \leq 400 \mathrm{~K}$ are

\begin{tabular}{|c|c|c|c|c|c|c|}
\hline & Ref. & $a_{0}[\AA]$ & $b_{0}[\AA]$ & $c_{0}[\AA]$ & $v_{0}\left[\AA^{3}\right]$ & $\Delta v_{0}[\%]$ \\
\hline \multicolumn{7}{|c|}{ PETN-I } \\
\hline$a$ & $\#$ & 9.33 & 9.33 & 6.66 & 579.75 & $-1.80 \%$ \\
\hline$b$ & {$[17]$} & 9.868 & 9.868 & 6.925 & 674.3 & 14.22 \\
\hline$c$ & {$[1]$} & 9.617 & 9.612 & 6.826 & 630.99 & 6.88 \\
\hline$d$ & [17] & 9.439 & 9.439 & 6.762 & 602.4 & 2.04 \\
\hline e & [18] & 9.2506 & 9.2506 & 6.6777 & 571.4369 & -3.21 \\
\hline$f$ & {$[5]$} & 9.3348 & 9.3348 & 6.6500 & 578.7628 & -1.97 \\
\hline$g$ & [3] & 9.38 & 9.38 & 6.71 & 590.375 & 0 \\
\hline$g$ & [2] & 9.383 & 9.383 & 6.711 & 590.841 & - \\
\hline \multicolumn{7}{|c|}{ PETN-II } \\
\hline$a$ & \# & 13.09 & 13.29 & 6.73 & 1170.79 & -4.39 \\
\hline$g$ & [3] & 13.29 & 13.49 & 6.83 & 1224.50 & 0 \\
\hline
\end{tabular}

$$
\begin{aligned}
& V_{\text {PETN-I }}=560.1598+0.0204 T \\
& \quad+1.5122 \times 10^{-4} T^{2}, \\
& a_{\text {PETN-I }}=9.2161+1.9292 \times 10^{-4} T \\
& \quad+6.4549 \times 10^{-7} T^{2}, \\
& c_{\text {PETN-I }}=6.5727+1.7648 \times 10^{-4} T \\
& \quad+3.9721 \times 10^{-7} T^{2}, \\
& V_{\text {PETN-II }}=1124.0293+0.0892 T \\
& \quad+2.4015 \times 10^{-4} T^{2}, \\
& a_{\text {PETN-II }}=12.9131+3.6923 \times 10^{-4} T \\
& \quad+8.4262 \times 10^{-7} T^{2}, \\
& b_{\text {PETN-II }}=13.1048+3.9710 \times 10^{-4} T \\
& \quad+8.1288 \times 10^{-7} T^{2}, \\
& c_{\text {PETN-II }}=6.6360 \\
& \quad+1.8995 \times 10^{-4} T+4.3411 \times 10^{-7} T^{2},
\end{aligned}
$$

TABLE I

Calculated equilibrium lattice parameters at ambient conditions compared with theoretical and experimental values obtained with different methods. "\#" — present work.

${ }^{a}$ NPT-MD; ${ }^{b}$ USP-PW91: ultrasoft pseudopotentials within generalized gradient approximation PerdewWang91 functional; ${ }^{c}$ PAW-PBE: projected augmented wave function pseudopotential with Perdew-BurkeErnzerhof functional; ${ }^{d}$ B3LYP $/ 6-31 \mathrm{G}^{* *}$ : B3LYP functional with 6-31G** Gaussian basis set; ${ }^{e} \mathrm{HF} / \mathrm{STO}-3 \mathrm{G}$ : Hartree-Fock method with the STO-3G basis set; ${ }^{f} \mathrm{MP}$ : molecular packing; ${ }^{g}$ experiment.

\subsection{Equation of state}

The equation of state, which is governed by molecular and intermolecular interaction in molecular crystal, 

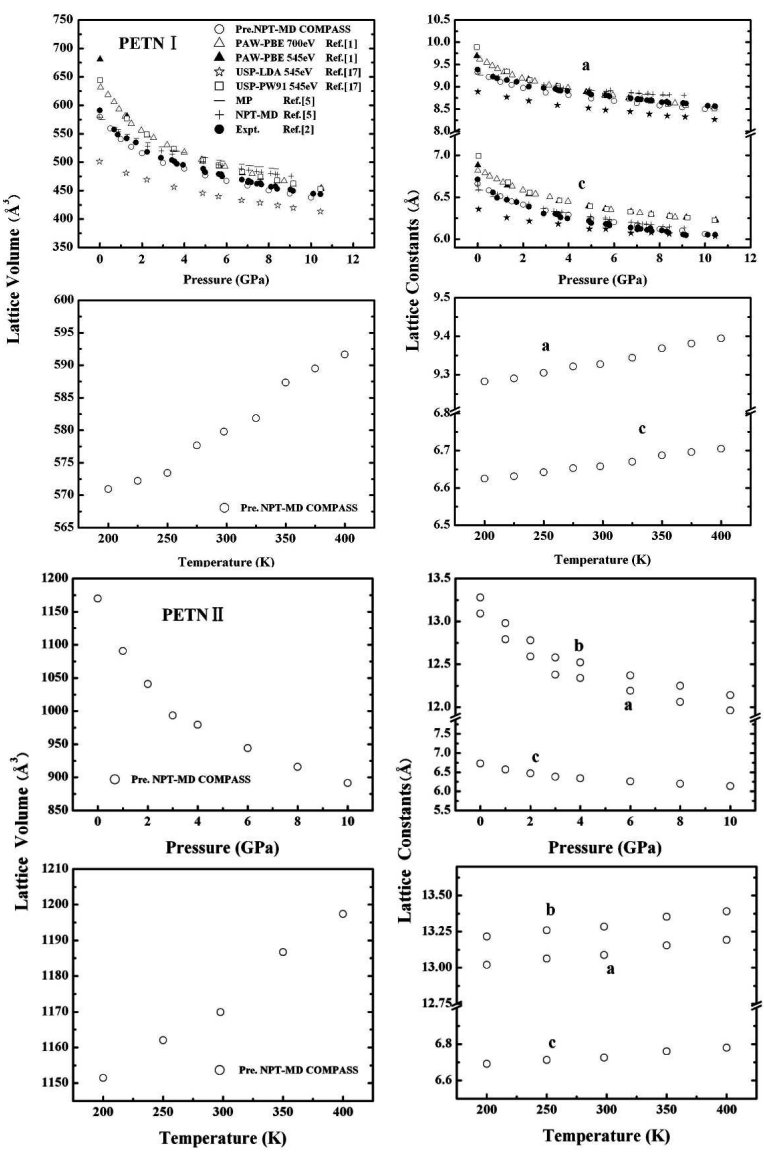

Fig. 3. Variation of cell volume and lattice constants with pressure at $298 \mathrm{~K}$, and with temperature at the standard pressure.

can give us excellent opportunity to link the microscopic and macroscopic properties of PETN. We plot the variation of lattice parameters under pressure or temperature, shown in Fig. 3. Due to the poor description of van der Waals interaction, which plays an important role in PETN molecular crystal, the DFT calculations significantly overestimate the cell volume at low pressure. But the DFT theoretical lattice parameters are closer to the experimental results with increase of pressure $[1,17]$. In contrast, the molecular simulations within the rigid-molecule approximation and molecular packing calculations achieve good agreement with experimental data at low pressure [5]. But the discrepancy increases with increase of pressure. The similarities of the results suggest that these potentials are not suitable for PETN under high pressure $[2,5]$. These methods cannot well predict the cell parameters at high pressure. Our calculated NPT-MD results with COMPASS force field can effectively improve the accuracy of the simulations in the experimental range of pressure [2].

There are several different formulations of EOS. Here, we fit the pressure-volume $(P-V)$ data of PETN to the Birch-Murnaghan EOS [19] and the Murnag- han EOS [20], respectively. The third-order BirchMurnaghan EOS can be written as

$$
P=3 B_{0} f_{E}\left(1+2 f_{E}\right)^{5 / 2}\left(1+3 / 2\left(B_{0}^{\prime}-4\right) f_{E}\right),
$$

where $f_{E}$ is written as $f_{E}=\left[\left(V_{0} / V\right)^{2 / 3}-1\right] / 2$ and $V_{0}$ is equilibrium lattice volume at ambient conditions. The Murnaghan EOS is written in the form of

$$
P=B_{0} / B_{0}^{\prime}\left[\left(V_{0} / V\right)^{B_{0}^{\prime}}-1\right] \text {. }
$$

Fitting the $P-V$ data to the EOS yields the bulk modulus $B_{0}$ and its first pressure derivative $B_{0}^{\prime}$ of PETN-I, as listed in Table II, together with other experimental and theoretical data. Using the Birch-Murnaghan EOS, our calculated $B_{0}$ and $B_{0}^{\prime}$ show excellent agreement with those of experiments [2]. They are better than those calculated by Conroy et al. [1]. In addition, using the Murnaghan EOS, our calculated $B_{0}$ and $B_{0}^{\prime}$ should be better than those calculated by Sorescu et al. [5] or Zaoui and Sekkal [11]. The reason is that our pressure-volume points approach experiments much better. We also predict the $B_{0}$ and $B_{0}^{\prime}$ of PETN-II using the two forms of EOS.

\section{TABLE II}

The calculated bulk modulus $B_{0}[\mathrm{GPa}]$ and its first pressure derivative $B_{0}^{\prime}$ of PETN at ambient conditions compared with theoretical and experimental values.

\begin{tabular}{c|c|c|c|c}
\hline \hline EOS & Ref. & Method & $B_{0}$ & $B_{0}^{\prime}$ \\
\hline \multicolumn{5}{c}{ PETN-I } \\
\hline BM & $\#$ & NPT-MD & 9.9 & 10.8 \\
BM & {$[1]$} & DFT/PAW-PBE & 9.1 & 8.3 \\
BM & {$[2]$} & exp. & 9.9 & 11.0 \\
M & $\#$ & NPT-MD & 12.0 & 6.8 \\
M & {$[5]$} & NPT-MD & 14.09 & 10.39 \\
M & {$[11]$} & NVT-MD & 9.16 & 2.59 \\
\hline \multicolumn{5}{c}{ PETN-II } \\
\hline BM & $\#$ & NPT-MD & 6.0 & 21.1 \\
M & $\#$ & NPT-MD & 9.6 & 8.4
\end{tabular}

\subsection{Elastic and mechanical properties}

The elastic constants of solids provide a link between the mechanical and dynamical behaviors of crystals, and give important information concerning the nature of the forces operating in solids. Computer simulation has remarkable advantage with its safe, effective, and economical capability to comprehend the properties of highly energetic materials under extreme conditions. The good predictions of lattice parameters and EOS above indicate that the MD simulations with COMPASS force field can give good description of interatomic binding forces and intermolecular interaction in PETN crystal. As shown in Table III, several experimental efforts have been done on the elastic constants of PETN-I. The theoretical calculations have not given all the elastic constants of PETN-I. Our NPT-MD calculations agree well with the experiments. The good agreement of experimental and theoretical elastic constants and EOS of PETN-I proves the 
validity and accuracy of the calculation for PETN. The elastic modulus of PETN under temperature at the standard pressure or under pressure at $298 \mathrm{~K}$ are calculated and listed in Table IV. We also plot the variation of bulk modulus $B$, shear modulus $G$, the Young modulus $E$, and the Poisson ratio $\nu$ as a function of the pressure or temperature, as shown in Fig. 4. The quantitative descriptions of the mechanical properties as a function of the pressure (for $P \leq 10 \mathrm{GPa}$ ) or temperature for $200 \mathrm{~K} \leq T \leq 400 \mathrm{~K}$ for PETN-I are

$$
\begin{aligned}
& B_{H}=8.537+6.376 P-0.082 P^{2}, \\
& G_{H}=4.863+1.019 P-0.014 P^{2}, \\
& E=12.365+3.125 P-0.054 P^{2}, \\
& \nu=0.276+0.033 P-0.002 P^{2}, \\
& B_{H}=13.8336-0.0190 T+6.5766 \times 10^{-6} T^{2}, \\
& G_{H}=5.9583-0.0036 T-1.4592 \times 10^{-7} T^{2}, \\
& E=15.6320-0.0110 T-4.2404 \times 10^{-7} T^{2}, \\
& \nu=0.3106-1.1301 \times 10^{-4} T-1.2163 \times 10^{-7} T^{2} .
\end{aligned}
$$

The predicted physical properties under temperature and pressure can provide powerful guidelines for the engineering application.
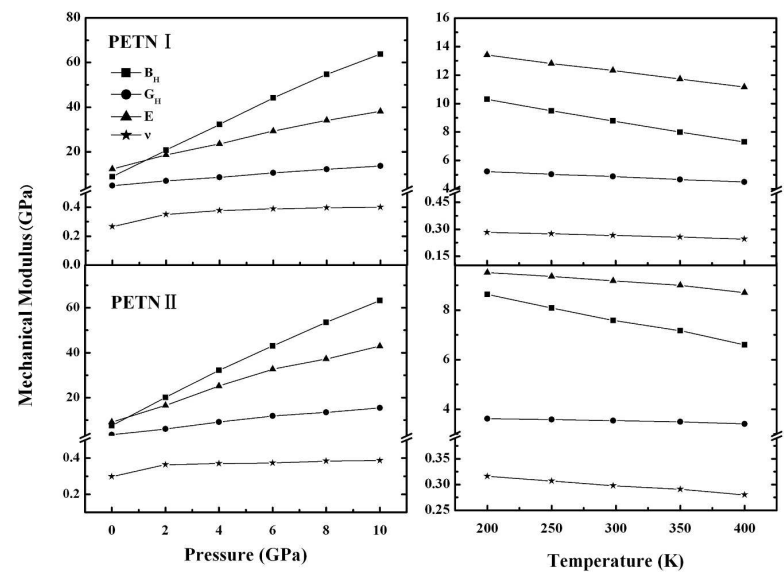

Fig. 4. The calculated pressure or temperature dependence of bulk modulus $B_{H}$ (squares), shear modulus $G_{H}$ (circles), the Young modulus $E$ (triangles), and the Poisson ratio $\nu$ (stars) for PETN.

\section{Conclusions}

Molecular dynamics simulations have been employed to study the lattice parameters, EOS, elastic constants, and mechanical modulus under pressure and temperature. The calculated equilibrium lattice parameters of PETN at ambient conditions are in good agreement with the experiments. We predict the lattice parameters of PETN under pressure and temperature. The bulk modulus $B_{0}$ and its pressure derivative $B_{0}^{\prime}$ are obtained by fitting the pressure--volume points to the Birch-Murnaghan EOS and Murnaghan EOS, respectively. The elastic constants of PETN under pressure and temperature are pre-
TABLE III

The calculated elastic constants [GPa] of PETN at ambient conditions compared with theoretical and experimental values.

\begin{tabular}{|c|c|c|c|c|c|c|c|c|c|}
\hline$P / T$ & $C_{11}$ & $C_{22}$ & $C_{33}$ & $C_{44}$ & $C_{55}$ & $C_{66}$ & $C_{12}$ & $C_{13}$ & $C_{23}$ \\
\hline \multicolumn{10}{|c|}{$P[\mathrm{GPa}]$} \\
\hline $10^{-4}$ & 16.62 & & 10.46 & 5.44 & & 5.90 & 6.67 & 6.09 & \\
\hline 2 & 40.22 & & 21.92 & 4.73 & & 10.04 & 11.32 & 16.83 & \\
\hline 4 & 61.48 & & 33.72 & 4.54 & & 13.52 & 16.07 & 27.24 & \\
\hline 6 & 80.62 & & 47.01 & 5.25 & & 17.52 & 22.48 & 37.93 & \\
\hline 8 & 99.25 & & 56.95 & 5.61 & & 21.96 & 28.03 & 47.75 & \\
\hline 10 & 113.60 & & 64.96 & 6.72 & & 23.29 & 34.56 & 56.57 & \\
\hline \multicolumn{10}{|c|}{$T[\mathrm{~K}]$} \\
\hline 200 & 19.36 & & 11.92 & 5.51 & & 6.56 & 7.54 & 7.44 & \\
\hline 250 & 17.84 & & 11.13 & 5.47 & & 6.20 & 7.06 & 6.72 & \\
\hline 298 & 16.62 & & 10.46 & 5.44 & & 5.90 & 6.67 & 6.09 & \\
\hline 350 & 15.23 & & 9.69 & 5.38 & & 5.52 & 6.18 & 5.38 & \\
\hline 400 & 14.06 & & 8.98 & 5.29 & & 5.20 & 5.74 & 4.78 & \\
\hline \multicolumn{10}{|c|}{$P[\mathrm{GPa}]$} \\
\hline $10^{-4}$ & 9.88 & 19.13 & 9.63 & 3.46 & 4.98 & 2.90 & 5.06 & 4.69 & 7.16 \\
\hline 2 & 24.74 & 36.83 & 20.97 & 12.74 & 8.17 & 3.79 & 17.99 & 16.21 & 19.18 \\
\hline 4 & 39.26 & 49.83 & 33.37 & 20.47 & 11.59 & 8.81 & 29.40 & 27.51 & 30.22 \\
\hline 6 & 51.27 & 61.98 & 45.56 & 25.12 & 14.90 & 14.55 & 39.51 & 37.39 & 40.68 \\
\hline 8 & 61.62 & 73.17 & 57.30 & 26.59 & 17.12 & 18.41 & 49.32 & 47.34 & 50.63 \\
\hline 10 & 74.77 & 84.48 & 68.59 & 27.58 & 19.13 & 21.56 & 57.41 & 55.75 & 60.02 \\
\hline \multicolumn{10}{|l|}{$T[\mathrm{~K}]$} \\
\hline 200 & 10.63 & 21.48 & 10.65 & 4.25 & 5.31 & 2.33 & 6.26 & 5.52 & 8.41 \\
\hline 250 & 10.20 & 20.27 & 10.12 & 3.82 & 5.15 & 2.63 & 5.62 & 5.09 & 7.74 \\
\hline 298 & 9.88 & 19.13 & 9.63 & 3.46 & 4.98 & 2.90 & 5.06 & 4.69 & 7.16 \\
\hline 350 & 9.66 & 18.08 & 9.19 & 3.17 & 4.83 & 3.13 & 4.57 & 4.36 & 6.63 \\
\hline 400 & 9.45 & 16.61 & 8.55 & 2.82 & 4.60 & 3.39 & 3.94 & 3.94 & 5.91 \\
\hline
\end{tabular}

\begin{tabular}{c|c|c|c|c|c|c|c|c|c|c}
\hline \hline Ref. & Meth. & $C_{11}$ & $C_{22}$ & $C_{33}$ & $C_{44}$ & $C_{55}$ & $C_{66}$ & $C_{12}$ & $C_{13}$ & $C_{23}$ \\
\hline \multicolumn{10}{c}{ PETN-I } \\
\hline$\#$ & NPT-MD & 16.62 & & 10.46 & 5.44 & & 5.90 & 6.67 & 6.09 & \\
{$[9]$} & exp. & 17.12 & & 12.18 & 5.03 & & 3.81 & 6.06 & 7.98 & \\
{$[8]$} & exp. & 17.22 & & 12.17 & 5.04 & & 3.95 & 5.44 & 7.99 & \\
{$[1]$} & PAW-PBE & 18.3 & 18.5 & 14.2 & & & & & & \\
{$[10]$} & NVT-MD & 11.8 & & & 2.8 & & & 7.8 & & \\
\hline \multicolumn{10}{c}{ PETN-II } \\
\hline$\#$
\end{tabular}

BM: Birch-Murnaghan equation of state,

M: Murnaghan equation of state

TABLE IV

The calculated elastic modulus (GPa) of PETN under temperature at the standard pressure or under pressure at $298 \mathrm{~K}$

dicted. Our molecular dynamics simulated elastic constants of PETN at ambient conditions. The elastic constants are in good agreement with the experimental data. The predicted elastic constants of two polymorphs under pressure and temperature should be accurate and provide powerful guidelines for further experimental measurement. In terms of the Voigt-Reuss-Hill approximation, the mechanical modulus is obtained. The good agreement between molecular dynamics results and experimental data prove that the molecular dynamics simulations with COMPASS force field can give good description of interatomic binding forces and intermolecular interaction in PETN crystal. 


\section{Acknowledgments}

The authors would acknowledge the China Postdoctoral Science Foundation funded project (Grant No. 2014M562336), National Natural Science Foundation of China (Grant No. 11664002, 11504035, and 11504304), Project of State Key Laboratory Breeding Base of Nuclear Resources and Environment (Grant No. NRE1321), the Science and Technology Project of Department of Education of Jiangxi Province (Grant No. GJJ14498).

\section{References}

[1] M.W. Conroy, I.I. Oleynik, S.V. Zybin, C.T. White, Phys. Rev. B 77, 094107 (2008).

[2] B. Olinger, P.M. Halleck, H.H. Cady, J. Chem. Phys. 62, 4480 (1975).

[3] H.H. Cady, A.C. Larson, Acta Crystallogr. B 31 , 1864 (1975).

[4] E.F.C. Byrd, B.M. Rice, J. Phys. Chem. C 111, 2787 (2007).

[5] D.C. Sorescu, B.M. Rice, D.L. Thompson, 6783, J. Phys. Chem. B 103, (1999).

[6] H. Sun, J. Phys. Chem. B 102, 7338 (1998).
[7] J.J. Dick, J. Appl. Phys. 81, 601 (1997).

[8] Y.A. Gruzdkov, Y.M. Gupta, J. Phys. Chem. A 104, 11169 (2000).

[9] J.M. Winey, Y.M. Gupta, J. Appl. Phys. 90, 1669 (2001).

[10] B. Sun, J.M. Winey, N. Hemmi, Z.A. Dreger, K.A. Zimmerman, Y.M. Gupta, D.H. Torchinsky, K.A. Nelson, J. Appl. Phys. 104, 073517 (2008).

[11] A. Zaoui, W. Sekkal, Solid State Commun. 118, 345 (2001).

[12] M. Nieger, J. Lehmann, private communication, 2002.

[13] Materials Studio.

[14] H.J.C. Berendsen, J.P.M. Postma, W.F.van Gunsteren, A. DiNola, J.R. Haak, J. Chem. Phys. 81, 3684 (1984).

[15] H.C. Andersen, J. Chem. Phys. 72, 2384 (1980).

[16] P.P. Ewald, Ann. Phys. 64, 253 (1921).

[17] W.F. Perger, J. Zhao, J.M. Winey, Y. Gupta, Chem. Phys. Lett. 428, 394 (2006).

[18] H.V. Brand, J. Phys. Chem. B 109, 13668 (2005).

[19] F. Birch, Phys. Rev. 71, 809 (1947).

[20] F.D. Murnaghan, Am. J. Math. 49, 235 (1937). 